\title{
Research on High Frequency Signal Anti-interference and Corresponding Applications on the Design and Implementation of Cheating Signal Detector
}

\author{
Kun Hou ${ }^{1, a}$, Yongfeng Ren ${ }^{1, a}$ and Guoxin Liu $^{1, a}$ \\ ${ }^{1}$ Institute of Information and Communication Engineering, \\ North University of China, Taiyuan 030051, China \\ ahoukun666666@126.com
}

Keywords: Signal Anti-interference, Cheating Signal Detector, High Frequency, Hardware Design.

\begin{abstract}
With the bursting and rapid development of computer science and technology, the need for designing signal anti-interference devices is urgent. Therefore, we conduct research on high frequency signal anti-interference and corresponding applications on the design and implementation of cheating signal detector in this research paper. We firstly introduce the basic principles and concepts of Fractional Fourier transform which is the generalized form of Fourier transform which could considers the same frequency interference cellular scene forming the filter coefficient is known, and channel fading coefficient can be estimated by training sequence. Later, through analyzing the hardware organization we design the hardware portion of the proposed system with PCB layout description and visualized figures. In the final part, we conclude our research and set of our prospect. In the future, we plan to test more circuit structures to optimize the current pattern.
\end{abstract}

\section{Introduction}

Linear frequency modulation interference is a typical broadband non-stationary interference, the current study its anti-interference are mainly concentrated in the interference rejection of spread spectrum signal, the corresponding method although LFM interference can be suppressed to a certain extent, but also damage the communication signal. In contrast to the mechanism of interference suppression, single channel blind source separation mainly adopts one letter fanaticism date processing method, disturbance of the received signal of single road separation analysis, estimate, and ingredients which will not damage under the premise of communication signals, maximum separation of communication signals and disturbance, achieve the goal that guarantee the normal communication, and the one letter blind source separation method can handle the spread spectrum and spread spectrum communication signal, therefore, its broad prospect of application is attracting more and more researchers into the study work. For single channel blind signal separation problem at present, there are three main kinds of research train of thought: one is by constructing multi-channel converting single channel blind source separation method of general positive definite cases of blind source separation problem, the shortcomings of this method is sensitive to noise, and that there are when the signal frequency deviation and phase deviation separation effect is not good; Second, by using the principle of transform domain, looking for can reflect differences between signal and interference of spectral domain, and then in the spectral domain structure filter, the difficulty of this method is that not all signals can reflect differences in the spectral domain,; Third, according to the principle of the Bayesian filtering, the blind source separation into state space model of the communication code and the joint estimation problem of unknown parameters, separation of communication signals by sequential estimation and interference.

Through the survey of cheating in existing equipment communications equipment, the main rubber cheating equipment mainly include rare earth headphones and text messages. Rare earth also called magnetic resonance headsets, the use of magnetic material in magnetic field by the principle of magnetic effect, by hiding in the jacket pocket or a magnetic field coil winding in the waist, collar, promote the rare earth magnetic vibrations in hidden in the ear canal. Cheating in the equipment 
receiving equipment mainly has two kinds: intercom and special receiver. Radio frequency band is wide, from the point of cheating in calendar year confiscated equipment, amateur band, public space, and has a professional band, the frequency range from $400 \mathrm{MHZ}$ to $800 \mathrm{MHZ}$. The special receiver generally works in around $200 \mathrm{MHZ}$ frequencies. SMS rubber is cheating equipment appeared in recent years, usually by integration of higher short-range wireless digital transmission chip answers, and through the LCD display. In order to disguise, this kind of equipment will also be disguised as a pen, pen bag, beverage bottle, watches, etc. Cheating in the communications equipment signal the possibility of wide frequency band, and digital signal are burst communications, not easy to be detection and interference. Aimed at this situation, it is necessary to design a wide frequency range and can undertake large range of suppressing interference and frequency hopping frequency sweep jammer, cheating in interference signal, guarantee the seriousness and fairness of the exam.

In this paper, we conduct research on high frequency signal anti-interference and corresponding applications on the design and implementation of cheating signal detector. We firstly introduce the foundation knowledge and prior research on signal transmission and anti-interference with theoretical analysis then through designing hardware circuits, we implement the corresponding device for detecting the cheating signal. Finally, we test our device with simulation result. The experimental result verifies the effectiveness and feasibility of our method.

\section{Foundations of Signal Transmission and Anti-interference}

Fractional Fourier transform of signals. Fractional Fourier transform is a generalized form of Fourier transform, can be interpreted as: in the time-frequency plane, the signal around the origin of the coordinate axes counterclockwise rotation at any Angle of a kind of representation. Essence is a kind of unified time-frequency transform, its size and FFT computation of the algorithm is quite quickly. Fractional Fourier transform can be represented as integral form:

$$
\begin{aligned}
& F^{p} f(u)=\int_{-\infty}^{+\infty} f(t) K_{p}(u, t) d t \\
& A_{\alpha}=\exp [-j \pi \operatorname{sgn}(\sin \alpha) / 4+j \alpha / 2] /|\sin \alpha|^{1 / 2}
\end{aligned}
$$

The corresponding visual description of the transform is shown in the figure 1.

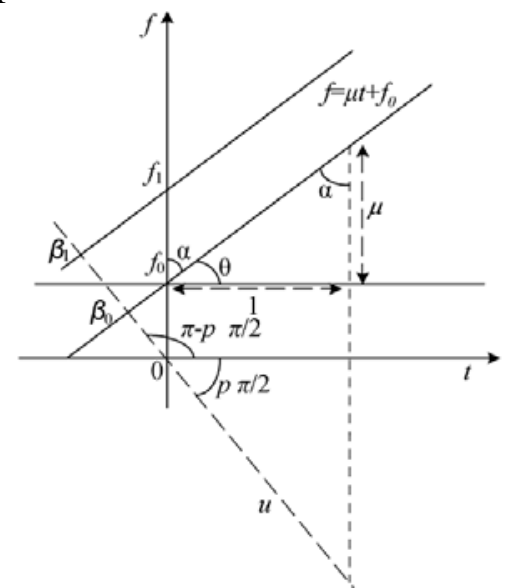

Fig. 1The Visual Description of the Transformation

For in the case of unknown parameters with channel mixed signal time delay estimation method, its performance is influenced by two signal carrier frequency difference, carrier frequency difference is smaller, the worse performance, so the algorithm is not suitable for the same frequency interference. Considering the same frequency interference cellular scene, forming the filter coefficient is known, and channel fading coefficient can be estimated by training sequence, therefore this section on certain assumptions do to relax, that forming filter coefficient and two way signal fading coefficient, time delay estimation method is discussed later in this paper. 
The State Space Model of Single Channel Disturbance Signal. In a digital communication system, single-channel receiver to receive the signal and disturbance of time-varying amplitude of LFM interference signal, the baseband signal after frequency conversion can be expressed as:

$$
y(t)=h_{1} e^{j\left(2 \pi \Delta f_{1} t+\Delta \theta\right)} \sum_{n=-L}^{L} a_{n} g(t-n T-\tau)+v(t)
$$

According to characteristic of communication element finite character set, in the code and the joint estimation of parameters, combined with the evolution strategy of genetic algorithm, can improve the estimation precision of particle filter and reduce the convergence time. Its main idea is to communication code and unknown parameters as chromosome samples, the corresponding weights of each sample as a fitness function, the standard particle filter tracking algorithm of resampling improvement for the process of selection, crossover and mutation of genetic algorithm, in order to solve the problem of particle degradation and exhausted. Through the selection and crossover operation makes the offspring sample for parent there are both inheritance and change, and through various dyeing samples corresponding to the size of the likelihood function makes the offspring of the change of the parent towards the optimal evolution to gain better anti-interference result.

\section{Design and Implementation of Proposed System}

Hardware Design and Description. DDS and the use of high integration digital waveform synthesizer AD9854, the chip on chip integrates two way orthogonal D/A converter, high speed, high performance through digital programming can output two synthetic signals I, Q. AD9854 DDS core has 48 frequency resolution (under $300 \mathrm{~m}$ system clock, the frequency resolution can reach $1 \mathrm{uHZ}$ ). AD9854 allows output signal frequency up to $150 \mathrm{MHZ}$, and digital modulation output frequency is $100 \mathrm{MHZ}$. Through internal high-speed comparator sine wave is converted to a square wave output, can be used as a convenient clock generator. Under the drive of high stability of clock, AD9854 will produce a highly stable frequency, phase and amplitude of programmable sine and cosine signals, can be used as the local oscillator used in communication, radar, etc. We show the sample circuit in the figure 2. More related circuit will be released on the future research.

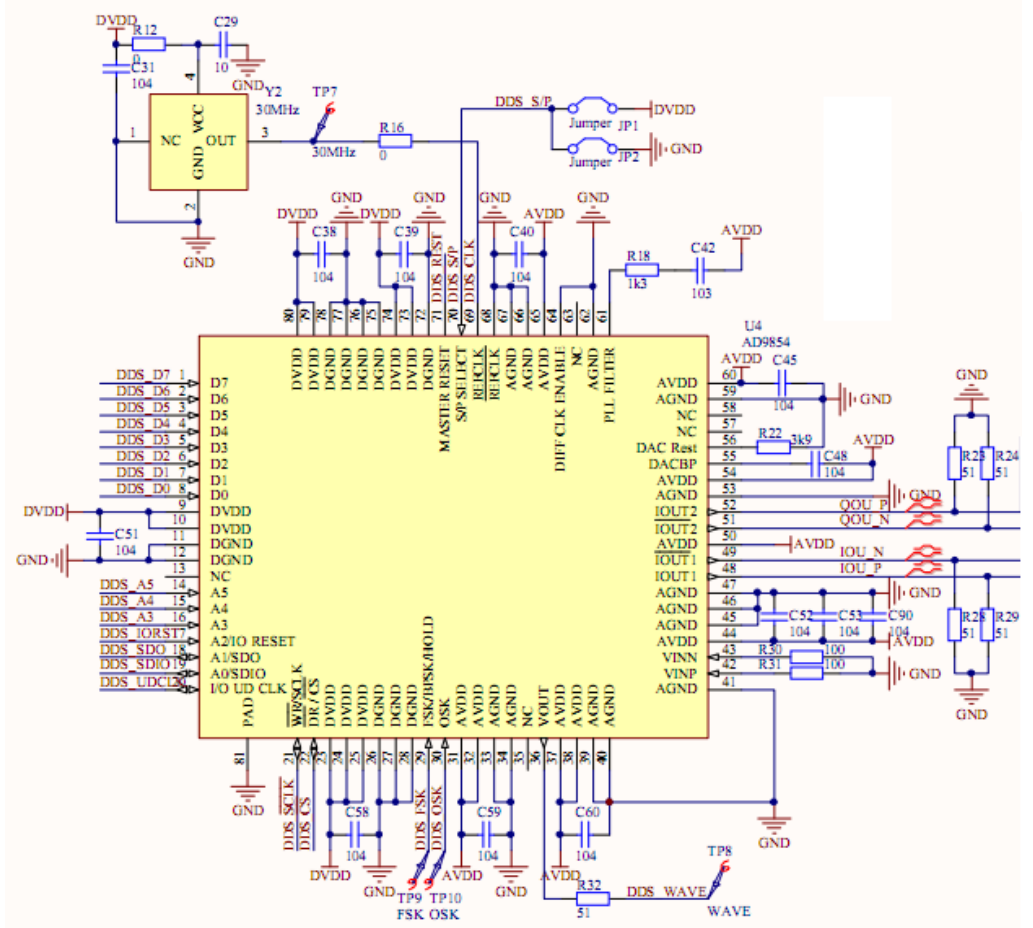

Fig. 2The Sample Circuit and Structure of the Hardware Portion

The Design of the PCB Board. Due to the high frequency circuit requires complete ground plane, to shield interference and to provide a complete reference ground plane micro-strip line, so the four layer board design. Running from top to bottom, respectively is the top layer, power line, formation 
and the underlying layer. Including the top and the bottom as the main line layer, layer according to the digital power supply, analog power supply and voltage different do break up, strata do not break up, the whole system altogether. The underlying layout as shown in figure 3 has components that are mainly micro control unit, a quadrature modulator, broadband gain module and power, etc. On the orthogonal frequency converter input is strict with phase, when I, Q signal phase delay will cause different road mix noise, so as far as possible when the layout is symmetrical. In order to reduce plate RF signal radiation, DDS to quadrature modulator, PLL to orthogonal signal lines of the modulator adopts differential walk the line, in the concrete implementation using Altium Designer wiring software function of the difference of guarantee differential linear features.

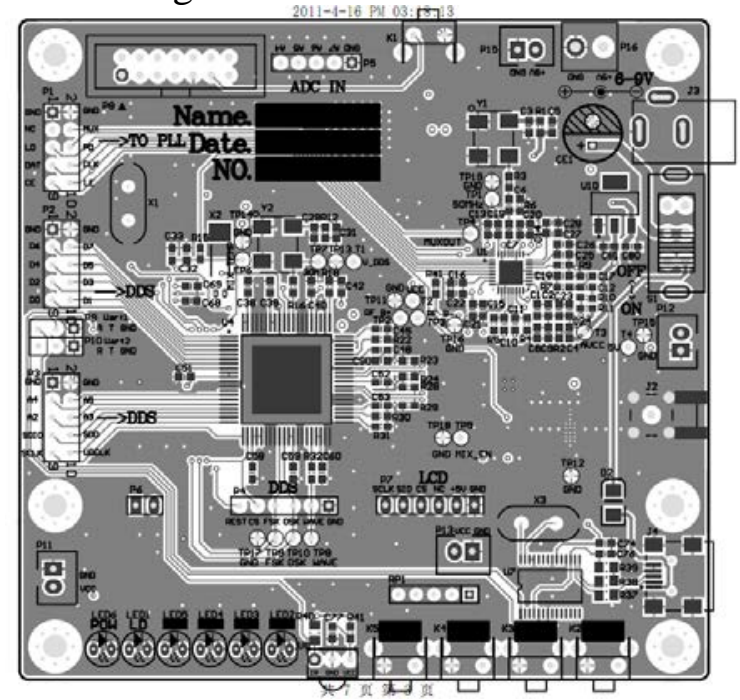

Fig. 3The Layout of the PCB Board for the System

\section{Summary}

In this paper, we take research on the design and implementation of high frequency signal anti-interference system. We firstly introduce the foundation knowledge and prior research on signal transmission and anti-interference with theoretical analysis then through designing hardware circuits, we implement the corresponding device for detecting the cheating signal. Finally, we test our device with simulation and experimental result. In the future, we decide to directly controlled by triangle wave VCO frequency sweep frequency sweep signal is generated, although the frequency control is not accurate, but can greatly reduce the cost and further more, we plan to conduct more mathematical analysis on the FFT transformation portion to obtain better test result.

\section{References}

[1] Chun-you, H. (2014). Anti-interference technology of professional wireless microphone on scene of large scale show. Entertainment Technology.

[2] Wang, L., Wang, H., Wong, K., \& Brennan, P. V. (2014). Minimax robust jamming techniques based on signal-to-interference-plus-noise ratio and mutual information criteria. Communications, IET, 8, 10, 1859 - 1867.

[3] Yang, P., Kang, L., \& Yue, L. (2014). A new approach for online identification of low frequency oscillation modes of power system based on the improved Hilbert-Huang transform. Transportation Electrification Asia-Pacific (ITEC Asia-Pacific), 2014 IEEE Conference and Expo (pp.1 - 4). IEEE.

[4] Bin, C., Xiang-yun, H., Dao-han, L., \& Yun-xia, Z. (2014). The development history and new progress of magnetic resonance sounding technique. Progress in Geophysics.

[5] Dao-cheng, Z., \& Zheng-cheng, Z. (2014). Research into transform domain anti-interference technology for shipboard navigation receiver. Shipboard Electronic Countermeasure. 